\title{
The Weight, Inflammation, Diet and Heart (WIDtH) Study Looking at Potential Primary Prevention of Coronary Artery Disease
}

\author{
Richard M Fleming ${ }^{1 *}$, Matthew R Fleming ${ }^{1}$, Gordon M Harrington ${ }^{2}$ and Tapan K Chaudhuri ${ }^{3}$ \\ ${ }^{1}$ FHHI-OmnificImaging-Camelot, El Segundo, CA, USA \\ ${ }^{2}$ Department of Psychology, University of Northern Iowa, Cedar Falls, IA, USA \\ ${ }^{3}$ Eastern Virginia Medical School, Norfolk, VA, USA \\ *Corresponding Author: Richard M Fleming, FHHI-OmnificImaging-Camelot, El Segundo, CA, USA.
}

Received: May 23, 2019; Published: June 13, 2019

DOI: $10.31080 /$ ASPS.2019.03.0309

\begin{abstract}
Background: Prior research has demonstrated improvement in coronary artery disease (CAD) when patients follow either a vegetarian or low to moderate fat diet, as long as caloric intake is limited to prevent weight gain. In individuals with known CAD research has demonstrated progression of disease in people following high protein, high fat, low carbohydrate diets. Numerous questions remain regarding the effect of several diets used by people to lose weight. These questions include how effectively do the diets result in weight loss, does the weight stay off once the diet is discontinued, what are the effects on multiple cardiovascular disease risk factors (CVDRFs) and what effect if any do these diets have on coronary blood flow. This study addresses those questions!.
\end{abstract}

Methods: One hundred and twenty men and women between 30 and 60 years of age were prospectively randomized into a $3 \times 2$ study to determine the effect of diet and a folate, B6/B12 supplement. Forty individuals followed a vegetarian diet, 40 followed a low to medium fat diet and 40 followed a low carbohydrate diet. Diets were adjusted to control for a caloric intake of 1500 - 1600 kilocalories per day (kcal/d). Half of the individuals in each group received foltx on a once daily basis. Participants were followed for 1 year and returned for re-evaluation at 6 weeks, 3, 6 and 12 months, and again 4 months after completion of the dietary program. Assessment included evaluation of respiratory quotient (RQ) to objectively evaluate diet, ketone strips of urine for the low carbohydrate group to further support maintenance of the low carbohydrate diet, weight (both pounds and kilograms), initial assessment of height, calculation of body mass index (BMI), lipids (total cholesterol/TC, low density lipoprotein cholesterol/LDL, high density lipoprotein cholesterol/HDL, TC/HDL ratio, very low density lipoprotein cholesterol/VLDL, triglycerides/TG), calculation of insulin resistance using TG/HDL ratios, determination of inflammation and thrombotic (inflammothrombotic) variables (c-reactive protein/ CRP, interleukin-6/IL-6, homocysteine/Hcy, fibrinogen/Fib, and lipoprotein (a)/Lp(a). Determination of coronary blood flow was made at baseline and again one-year later using myocardial perfusion imaging.

Results: Sixty two men and 58 women completed one year of dietary therapy with an average age of $43+/-8$ years and a BMI greater than 30. Participants lost between 24 and 42 pounds. Respiratory quotients and urinary ketones confirmed the subjective information provided by patients, viz. that they were in deed following the prescribed dietary regimens. Weight loss did not guarantee associated changes in cardiovascular disease risk factors (CVDRFs) or coronary blood flow. TC and LDL levels dropped significantly ( $\mathrm{p}<$ 0.001) while following the vegetarian or low to moderate fat diet with or without supplement. Initially, TC and LDL levels dropped on the low carbohydrate diet with or without supplement. This decreased stopped 3 months into the diet and then increased reaching initial values over the remainder of the study. Similar changes were seen for VLDL, TG and insulin resistance (TG/HDL). HDL levels increased for people on the vegetarian and low to moderate fat diets ( $p<0.005$ to 0.001$)$ with or without supplement. These changes were not seen on the low carbohydrate diet even though there were no differences in exercise regimen.

C-reactive protein decreased on the vegetarian diet with supplement $(\mathrm{p}<0.025)$ and the low to moderate fat diets with $(\mathrm{p}<$ $0.025)$ or without $(\mathrm{p}<0.005)$ supplement. Changes in CRP were not significant on the other diets. Interleukin 6 levels did not change on the low to moderate fat diet without supplement. Interleukin 6 decreased significantly $(\mathrm{p}<0.01)$ on the vegetarian diet with and without supplement and on the low to moderate fat diet with supplement. Interleukin 6 increased $(p<0.005)$ on the low carbohydrate diet without supplement more than with $(\mathrm{p}<0.05)$ supplement. Homocysteine levels decreased on both the both the vegetarian diet without supplement $(\mathrm{p}=\mathrm{NS})$ and with supplement $(\mathrm{p}<0.01)$ and the low to moderate fat diet without supplement $(\mathrm{p}$

Citation: Richard M Fleming., et al. "The Weight, Inflammation, Diet and Heart (WIDtH) Study Looking at Potential Primary Prevention of Coronary Artery Disease". Acta Scientific Pharmaceutical Sciences 3.7 (2019): 33-44. 
$<0.005)$ and with $(\mathrm{p}<0.001)$ supplement. Homocysteine levels increased $40.2 \%$ on the low carbohydrate diet without supplement but remained unchanged with the supplement. Minimal changes were noted in fibrinogen levels with the most pronounced changes occurring on the low carbohydrate diet. Lipoprotein (a) levels did not change appreciably except for the decrease seen with the low to moderate fat diet without $(\mathrm{p}<0.01)$ and with $(\mathrm{p}<0.005)$ supplement. These changes in CVDRFs were associated with improvement in coronary blood flow for those following the vegetarian and low to moderate fat diets. This was statistically $(p<0.025)$ significant when those on the low to moderate fat diet took the supplement. Coronary blood flow worsened for those on the low carbohydrate diet regardless of supplement use.

Conclusion: Weight loss occurs on low to moderate fat, low carbohydrate and vegetarian diets as long as caloric intake is accounted for. This weight loss is not necessarily associated with a reduction in CVDRFs or coronary blood flow. While there are initial improvements in lipids on the low carbohydrate diet, this is brief, lasting only the first few months, after which there is an increase in risk factors and an associated deterioration in coronary perfusion. Low to moderate fat and vegetarian diets are associated with reduction in CVDRFs (lipids, insulin resistance, and inflammothrombotic variables) and improvement in coronary blood flow. Following cessation of the monitoring, those on the vegetarian and low to moderate fat diet were able to keep weight off or continue to lose additional weight, while those on the low carbohydrate diet showed weight increase.

Keywords: FMTVDM ${ }^{\oplus}$; Heart Disease, Primary Prevention

\section{Introduction}

Changes in dietary and lifestyle patterns in recent years have resulted in an increased incidence of overweight and obesity problems in the United States and Europe. Problems resulting from this include hypertension, diabetes, certain forms of cancer and heart disease. Various diets have been proposed as useful for weight loss. To date however there is limited information available as to the effect that these diets have on heart disease. Early work [1] did not place people on diets alone, but looked at the effect of diet, medications and lifestyle changes. Work following this separated out the effect of diet versus drug treatment [2-4] but looked only at lipids. Since then it has become apparent that heart disease is an inflammatory disease $[5,6]$ caused by elevations in lipids as well as multiple inflammatory and thrombotic variables. Efforts to begin looking at reversal of heart disease by trying to reduce each of the cardiovascular disease risk factors (CVDRFs) showed mixed $[7,8]$ results depending upon the type of diet people were following. This research [8] was the first and so far only work to demonstrate the effect of low carbohydrate diets and coronary artery disease. These studies were limited because it did not place individuals on vitamin and mineral supplements and as such could not determine if there was a benefit by doing so. Other research looking at lowcarbohydrate diets [9] has suggested that such diets can produce weight loss, but excluded people whose cholesterol levels went up. In a review of that study [10], it was established the weight loss was a reflection of caloric reduction and not carbohydrate content. The study also had problems with patient retention and like two recently published studies $[11,12]$ placed patients on vitamin and mineral supplements. The studies therefore do not demonstrate the effect of diet alone, but diet with supplements while excluding those who showed worsening of lipid levels or problems staying on the diet. Research is beginning to look at several inflammatory variables [13-19] involved with heart disease, but this work has only just begun. Cumulatively the result of these prior studies have yield limited scientific insight into what truly happens when people follow dietary regimens, particularly when people do not take vitamin and mineral supplements prescribed by many to reduce deficiencies present in many of these diets.

Given the above questions, the WIDtH (Weight, Inflammation, Diet and Heart Disease) study was designed to answer the following: (1) What effect do vegetarian, low to moderate fat and low carbohydrate diets have on weight loss when caloric intake and exercise are equal? (2) Do people maintain weight loss after they have completed their diet program? (3) How do each of the diets affect lipids, inflammatory and thrombotic factors associated with heart disease and do vitamin supplements change this? (4) What happens to coronary blood flow after being on these diets?

\section{Methods \\ Subject recruitment and monitoring}

To be enrolled in the study, participants had to be between the ages of 30 and 59 with BMIs greater than 30 (obese). They had to be non-pregnant with no prior documented heart disease. Participants had to be on no medications including over the counter vitamins and supplements. Individuals with particular food allergies 
(e.g. gluten, dairy, peanuts, et cetera) were also prohibited from enrollment into the study. They had to be enrolled in no other studies and could not have diabetes, liver, renal, gastrointestinal disease or cancer. Participation in the study was voluntary, following human subject guidelines and informed consent.

Individuals were randomly assigned to one of three diets including vegetarian, low to moderate fat, and low carbohydrate diets. One half of all individuals assigned to each diet received a folate (2.5 mg), vitamin B6 (25 mg) and B12 (1 mg) supplement for 1 year. Subjects were followed for a total of 16 months. During that time they were evaluated upon entry (baseline) into the study, at 6 weeks, 3 months, 6 months and 1 year. They were reassessed four months after completion of the study.

\section{Dietary recommendations [7]}

Those following a vegetarian diet were asked to abstain from eating meat, which was defined as anything moving under its own power while alive (e.g. Beef, poultry, pork, fish, et cetera). Dairy products and eggs were also eliminated from the diets of this group.

Low to moderate fat diets including adjustment of fat intake to no more than 15 - $20 \%$ of the total caloric intake. No specific foods were eliminated on this diet as long as the total fat intake did not exceed this amount with no more than 5 grams of saturated fat consumed per day. During a typical day this would result in 20 - 25 grams of non-saturated fat and up to 5 grams of saturated fat.

Low carbohydrate diets were defined as diets where the consumption of carbohydrate did not exceed $25 \%$ of the recommended daily caloric intake, which equaled approximately 100 grams per day. The remainder of the caloric intake was divided between protein $(25 \%)$ and fat $(50 \%)$ consumption.

All individuals were instructed on caloric intake providing 1500 to $1600 \mathrm{kcal} /$ day, including instructions to avoid eating within 4 - 5 hours of going to sleep to avoid anabolism of these calories into fat deposits.

\section{Exercise regimen}

Individuals were instructed to follow one of three exercises (walking, bicycling or swimming) three times per week for thirty minutes per session. This could be done indoors or outdoors depending upon weather conditions and individual preference. Exercise was for time and not speed or distance. Stationary treadmills or bicycles could be used in the place of non-stationary sources of exercise depending upon personal preference.

\section{Anthropometric information}

Heights were taken upon entry into the study and were used to calculate BMI throughout the study. Weight was recorded in both pounds and kilograms during each of the assessments.

\section{Dietary compliance}

Information was collected regarding the specific dietary regimens followed by each subject. Confirmation was made objectively by determination of the respiratory quotient using a MedGraphics gas exchange program (BreezeEx v3.06) to determine the ratio of expired carbon dioxide $\left(\mathrm{VCO}_{2}\right)$ to oxygen $\left(\mathrm{VO}_{2}\right)$ in the resting state and urine sampling (ketone strips) of ketones.

\section{Fasting venous blood work}

During each of the evaluations fasting blood work was obtained for (1) depository variables (those which are associated with deposition of material within coronary arteries) including total cholesterol/TC, low-density lipoprotein cholesterol/LDL, high-density lipoprotein cholesterol/HDL, very low-density lipoprotein cholesterol/VLDL, and triglycerides/TG. From this insulin resistance (TG/ HDL) was estimated.

\section{Inflammothrombotic variables}

During the evaluations fasting venous blood was also obtained for C-reactive protein/CRP, interleuking-6/IL-6, homocysteine/ Hcy, fibrinogen/Fib and lipoprotein (a)/Lp(a).

\section{Coronary blood flow}

Myocardial perfusion imaging was completed at entry into the study and one year after following the recommended dietary and supplement protocol. Perfusion imaging was performed $[6,8,20]$ as previously established. Ischemia was determined based upon the severity and extent of perfusion limitation.

\section{Statistical analysis}

The overall age and sex of individuals enrolled in the study was determined following assessment of a BMI greater than 30. Results of this 3 (diets) x 2 (supplement vs. no supplement) study were then determined calculating mean $+/-$ standard deviations for comparison purposes using two-tailed t-test* using $\mathrm{p}<0.05$ for statistical significance [21]. Graphic display of BMI was made for each group plotted against time. 


\section{Results}

Demographic information

One hundred twenty men $(n=62)$ and women $(n=58)$ between 30 and $60(43.72+/-8.40)$ years of age were followed for 1 year while following one of three diets, with or without supplement. Four months later they were restudied to determine post diet response. No differences existed between groups for age or sex as shown in table 1.

\begin{tabular}{|c|c|c|c|}
\hline & Men & Women & Age ${ }^{*}$ \\
\hline Vegetarian without supplement & 12 & 8 & $42.55 / 9.27$ \\
\hline Vegetarian with supplement & 9 & 11 & $43.55 / 9.27$ \\
\hline $\begin{array}{c}\text { Low to moderate fat without } \\
\text { supplement }\end{array}$ & 10 & 10 & $44.05 / 9.82$ \\
\hline $\begin{array}{c}\text { Low to moderate fat with } \\
\text { supplement }\end{array}$ & 9 & 11 & $43.20 / 9.12$ \\
\hline $\begin{array}{c}\text { Low carbohydrate without } \\
\text { supplement }\end{array}$ & 12 & 8 & $45.10 / 6.82$ \\
\hline $\begin{array}{c}\text { Low carbohydrate with } \\
\text { supplement }\end{array}$ & 10 & 10 & $43.10 / 6.84$ \\
\hline
\end{tabular}

Table 1: Demographic information.

* mean/standard deviation

\section{Dietary confirmation}

Assessment of dietary changes consisted of two objective findings in addition to the subjective information provided by the individuals in the study. First the use of urinary ketone testing. Ketones were present in the urine of those following the low carbohydrate diet with or without supplement during the 12 months of dietary changes. Ketones ceased to be present during the four month follow up assessment. Those following the vegetarian and low to moderate fat diets showed no ketones on urinalysis. The changes in respiratory quotients (RQ) are shown in tables' $2 \mathrm{a}$ to $4 \mathrm{~b}$. There was no difference in $\mathrm{RQ}$ values between groups at the beginning of the study. Individuals on the vegetarian diet showed increased RQ values throughout the study when compared to baseline values. Those following the low to moderate fat diets demonstrated slightly but insignificantly increased RQ values. People on the low carbohydrate diets demonstrated reductions in RQ values consistent with lower carbohydrate (RQ of 0.84 and 0.83 ) intake. Those on the vegetarian diets taking supplements and those on the low carbohydrate diets with or without supplement intake showed changes in dietary patterns four months after completion of the dietary regimen.

\section{Coronary blood flow}

Early changes in coronary blood flow seen on myocardial perfusion imaging demonstrate reductions in coronary flow reserve. The cumulative results from all three epicardial artery beds are noted as the ischemia index (II). The baseline results were compared with findings at one year and are shown in tables' $2 \mathrm{a}$ to $4 \mathrm{~b}$. There were no statistical differences between blood flow amongst groups at the beginning of the study. Individuals following the vegetarian diet without supplement showed a $20.7 \%$ improvement in coronary flow. Those on the vegetarian diet who were taking the supplement demonstrated a $11.3 \%$ improvement. Those following the low to moderate fat diet without supplement revealed a $18.1 \%$ improvement while those taking the supplement showed a $43.6 \%$ improvement. This improvement was statistically $(\mathrm{p}<0.025)$ significant. Following one year on the low carbohydrate diet without supplement there was a $46.9 \%$ deterioration in coronary blood flow. Those following the low carbohydrate diet with supplement showed a less pronounced worsening (43.1\%) of blood flow.

\section{Anthropometric changes}

Changes in weight (pounds, kilograms) and basal metabolic index (BMI) are shown in tables' $2 \mathrm{a}$ to $4 \mathrm{~b}$. Changes in BMI are displayed in figure 1. There were no differences between groups at the beginning of the study and each individual in the study began with a BMI of greater than 30 . People on the vegetarian diet without supplement showed a weight loss of 30.2 (11.3\%) pounds or 13.5 kilograms during the first 12 months of the study. During this time the BMI dropped from an average of 42.0 to 37.2. This weight loss persisted four months later with a slight additional weight loss of 0.4 pounds or 0.2 kilograms. When those on the vegetarian diet also took the supplement they showed a 33.7 pound $(12.2 \%)$ or 14.9 kilogram weight loss during the first 12 months. The BMI decreased from 42.5 to 37.4. During the following four months, there was a 1.4 pound/0.7 kilogram weight gain. While these changes in weight approached, they did not reach, statistical significance.

The low to moderate fat diet without supplement resulted in a $33.7(12.2 \%)$ pound or 14.9 kilogram weight loss during the 12-month dietary change. The BMI decreased from an average of 42.5 to 37.4 during this time. Four months later there was further weight loss, with an additional 2.8 pounds/1.3 kilograms The BMI continued to decrease to 38.1 at the four-month follow up. This decrease in BMI was significant at $\mathrm{p}<0.05$. Those following the low to moderate fat diet with supplement began at an average of 267.1 


\begin{tabular}{|c|c|c|c|c|c|c|}
\hline & Baseline & $\mathbf{6}$ weeks & $\mathbf{3}$ months & $\mathbf{6}$ months & 1 year & 4 months after \\
\hline II & $0.087 / 0.049$ & NA & NA & NA & $0.069 / 0.047$ & NA \\
\hline RQ & $0.88 / 0.02$ & $0.90 / 0.02$ & $0.92 / 0.02$ & $0.92 / 0.03$ & $0.95 / 0.02$ & $0.95 / 0.03$ \\
\hline Pounds & $268.0 / 63.7$ & $261.0 / 62.9$ & $256.4 / 62.4$ & $248.1 / 61.8$ & $237.8 / 60.4$ & $237.4 / 59.3$ \\
\hline Kg & $121.6 / 29.1$ & $118.6 / 28.5$ & $116.6 / 28.4$ & $112.6 / 28.1$ & $108.1 / 27.5$ & $107.9 / 26.9$ \\
\hline BMI & $42.0 / 8.3$ & $40.9 / 8.1$ & $40.3 / 8.1$ & $38.9 / 8.0$ & $37.2 / 7.6$ & $37.2 / 7.6$ \\
\hline TC & $254.8 / 23.1$ & $242.0 / 21.9$ & $240.0 / 21.6$ & $231.9 / 20.4$ & $206.2 / 20.1$ & $201.2 / 32.9$ \\
\hline LDL & $172.7 / 24.1$ & $161.7 / 20.2$ & $161.7 / 20.2$ & $154.5 / 19.4$ & $132.9 / 16.6$ & $127.4 / 31.1$ \\
\hline HDL & $43.0 / 5.9$ & $44.4 / 7.6$ & $44.4 / 7.6$ & $46.2 / 6.6$ & $48.3 / 5.4$ & $49.2 / 4.9$ \\
\hline TC/HDL & $6.0 / 1.1$ & $5.6 / 0.9$ & $5.5 / 0.9$ & $5.1 / 0.8$ & $4.3 / 0.5$ & $4.1 / 0.8$ \\
\hline VLDL & $39.1 / 6.1$ & $35.9 / 4.6$ & $33.8 / 3.9$ & $31.1 / 3.7$ & $25.1 / 3.0$ & $24.5 / 3.6$ \\
\hline TG & $195.6 / 30.7$ & $179.6 / 23.1$ & $169.1 / 19.3$ & $155.4 / 18.3$ & $125.5 / 15.2$ & $122.3 / 18.0$ \\
\hline TG/HDL & $4.6 / 1.0$ & $4.1 / 0.8$ & $3.9 / 0.7$ & $3.4 / 0.6$ & $2.6 / 0.4$ & $2.5 / 0.5$ \\
\hline CRP & $0.81 / 1.46$ & $0.77 / 1.33$ & $0.61 / 1.6$ & $0.48 / 0.60$ & $0.28 / 0.14$ & $0.26 / 0.17$ \\
\hline IL-6 & $5.28 / 3.84$ & $5.11 / 3.45$ & $4.22 / 2.24$ & $3.51 / 1.99$ & $2.63 / 1.32$ & $2.11 / 1.25$ \\
\hline Hcy & $15.5 / 9.4$ & $16.2 / 8.3$ & $15.7 / 6.9$ & $14.4 / 6.9$ & $11.3 / 4.1$ & $15.5 / 19.9$ \\
\hline Fib & $331.5 / 66.4$ & $322.9 / 42.6$ & $322.3 / 33.6$ & $321.9 / 22.3$ & $321.4 / 20.3$ & $324.1 / 18.1$ \\
\hline Lp(a) & $26.6 / 13.2$ & $25.3 / 11.9$ & $24.0 / 11.5$ & $21.7 / 10.4$ & $20.4 / 9.7$ & $21.4 / 9.4$ \\
\hline
\end{tabular}

Table 2a: 1500-1600 kcal/day vegetarian diet without vitamin supplement*.

\begin{tabular}{|c|c|c|c|c|c|c|}
\hline & Baseline & $\mathbf{6}$ weeks & 3 months & $\mathbf{6}$ months & 1 year & 4 months after \\
\hline II & $0.080 / 0.040$ & NA & NA & NA & $0.071 / 0.033$ & NA \\
\hline RQ & $0.89 / 0.03$ & $0.92 / 0.02$ & $0.94 / 0.02$ & $0.94 / 0.03$ & $0.97 / 0.02$ & $0.92 / 0.03$ \\
\hline Pounds & $277.2 / 53.4$ & $270.4 / 53.2$ & $260.3 / 53.7$ & $251.1 / 54.5$ & $243.5 / 53.5$ & $244.9 / 53.0$ \\
\hline Kg & $125.6 / 24.1$ & $123.1 / 24.1$ & $118.3 / 24.4$ & $114.2 / 24.7$ & $110.7 / 24.3$ & $111.4 / 24.0$ \\
\hline BMI & $42.5 / 7.7$ & $41.3 / 7.6$ & $39.9 / 8.2$ & $38.6 / 8.2$ & $37.4 / 8.0$ & $37.5 / 8.2$ \\
\hline TC & $264.1 / 29.0$ & $249.1 / 30.5$ & $228.9 / 26.3$ & $224.0 / 23.9$ & $192.5 / 20.7$ & $189.8 / 18.0$ \\
\hline LDL & $181.7 / 27.5$ & $168.6 / 28.0$ & $149.9 / 23.7$ & $147.4 / 21.8$ & $119.0 / 20.0$ & $118.6 / 17.6$ \\
\hline HDL & $43.4 / 4.9$ & $44.3 / 4.0$ & $46.4 / 4.2$ & $47.0 / 4.3$ & $48.1 / 4.3$ & $47.0 / 4.2$ \\
\hline TC/HDL & $6.2 / 1.0$ & $5.7 / 0.8$ & $5.0 / 0.6$ & $4.8 / 0.6$ & $4.0 / 0.6$ & $4.1 / 0.5$ \\
\hline VLDL & $39.0 / 6.3$ & $36.2 / 5.9$ & $32.4 / 5.2$ & $29.5 / 5.2$ & $25.4 / 5.2$ & $24.2 / 4.6$ \\
\hline TG & $195.1 / 31.3$ & $181.2 / 29.6$ & $162.1 / 25.9$ & $147.4 / 26.0$ & $127.0 / 26.1$ & $121.2 / 23.0$ \\
\hline TG/HDL & $4.5 / 0.8$ & $4.1 / 0.7$ & $3.5 / 0.6$ & $3.2 / 0.6$ & $2.7 / 0.6$ & $2.6 / 0.5$ \\
\hline CRP & $2.34 / 3.55$ & $1.49 / 2.19$ & $0.76 / 1.24$ & $0.45 / 0.44$ & $0.28 / 0.22$ & $0.40 / 0.13$ \\
\hline IL-6 & $5.83 / 2.98$ & $5.11 / 2.91$ & $4.54 / 2.28$ & $4.15 / 1.76$ & $3.55 / 1.69$ & $3.34 / 1.31$ \\
\hline Hcy & $13.9 / 6.4$ & $12.0 / 5.9$ & $10.5 / 5.2$ & $10.0 / 4.1$ & $9.1 / 3.1$ & $9.0 / 3.1$ \\
\hline Fib & $327.4 / 72.1$ & $327.1 / 45.4$ & $327.4 / 28.3$ & $325.8 / 22.1$ & $329.1 / 18.2$ & $329.2 / 14.9$ \\
\hline Lp(a) & $23.4 / 12.0$ & $22.7 / 11.3$ & $21.4 / 10.8$ & $20.2 / 8.9$ & $19.0 / 8.5$ & $18.1 / 8.4$ \\
\hline
\end{tabular}

Table 2b: 1500-1600 kcal/day vegetarian diet with vitamin supplement*.

* values are presented as mean/standard deviation. 
The Weight, Inflammation, Diet and Heart (WIDtH) Study Looking at Potential Primary Prevention of Coronary Artery Disease

\begin{tabular}{|c|c|c|c|c|c|c|}
\hline & Baseline & $\mathbf{6}$ weeks & $\mathbf{3}$ months & $\mathbf{6}$ months & $\mathbf{1}$ year & 4 months after \\
\hline II & $0.083 / 0.055$ & NA & NA & NA & $0.068 / 0.041$ & NA \\
\hline RQ & $0.90 / 0.02$ & $0.93 / 0.01$ & $0.93 / 0.01$ & $0.92 / 0.01$ & $0.92 / 0.01$ & $0.92 / 0.01$ \\
\hline Pounds & $276.5 / 53.2$ & $268.9 / 53.2$ & $263.2 / 53.2$ & $254.8 / 53.0$ & $246.7 / 53.3$ & $243.9 / 53.3$ \\
\hline Kg & $125.7 / 24.2$ & $122.4 / 24.2$ & $119.6 / 24.2$ & $115.8 / 24.1$ & $112.2 / 24.2$ & $110.9 / 24.3$ \\
\hline BMI & $43.1 / 7.5$ & $42.0 / 7.2$ & $41.1 / 7.4$ & $39.7 / 7.3$ & $38.6 / 7.5$ & $38.1 / 7.5$ \\
\hline TC & $266.2 / 23.3$ & $239.5 / 22.4$ & $216.7 / 20.0$ & $196.2 / 21.3$ & $181.5 / 16.7$ & $170.9 / 28.6$ \\
\hline LDL & $183.4 / 21.3$ & $158.9 / 20.5$ & $136.5 / 17.4$ & $120.9 / 18.9$ & $108.2 / 14.1$ & $98.9 / 25.0$ \\
\hline HDL & $43.4 / 4.9$ & $45.4 / 5.3$ & $47.3 / 5.6$ & $47.0 / 3.7$ & $48.3 / 3.4$ & $49.0 / 4.1$ \\
\hline TC/HDL & $6.2 / 0.8$ & $5.3 / 0.6$ & $4.6 / 0.6$ & $4.2 / 0.5$ & $3.8 / 0.3$ & $3.5 / 0.5$ \\
\hline VLDL & $39.5 / 8.1$ & $35.3 / 5.2$ & $32.8 / 4.5$ & $28.2 / 4.1$ & $24.9 / 4.8$ & $23.0 / 4.5$ \\
\hline TG & $197.4 / 40.4$ & $176.3 / 26.2$ & $163.8 / 22.5$ & $141.0 / 20.6$ & $124.6 / 24.2$ & $115.1 / 22.4$ \\
\hline TG/HDL & $4.6 / 1.3$ & $3.9 / 0.8$ & $3.5 / 0.7$ & $3.0 / 0.4$ & $2.6 / 0.5$ & $2.4 / 0.5$ \\
\hline CRP & $0.71 / 1.02$ & $0.44 / 0.49$ & $0.24 / 0.14$ & $0.16 / 0.11$ & $0.09 / 0.09$ & $0.19 / 0.09$ \\
\hline IL-6 & $6.14 / 4.44$ & $5.77 / 3.94$ & $5.21 / 3.42$ & $4.86 / 3.12$ & $3.99 / 2.16$ & $3.85 / 2.03$ \\
\hline Hcy & $16.0 / 8.7$ & $14.0 / 6.9$ & $12.9 / 6.5$ & $10.9 / 5.1$ & $9.2 / 3.5$ & $9.2 / 3.3$ \\
\hline Fib & $326.7 / 61.4$ & $331.8 / 51.6$ & $338.7 / 42.2$ & $341.8 / 22.8$ & $333.9 / 20.8$ & $341.4 / 16.0$ \\
\hline Lp(a) & $22.8 / 10.1$ & $21.6 / 9.6$ & $19.4 / 8.7$ & $17.0 / 7.1$ & $15.0 / 6.6$ & $13.8 / 5.8$ \\
\hline
\end{tabular}

Table 3a: 1500-1600 kcal/day low to moderate fat diet without vitamin supplement*.

\begin{tabular}{|c|c|c|c|c|c|c|}
\hline & Baseline & $\mathbf{6}$ weeks & $\mathbf{3}$ months & $\mathbf{6}$ months & 1 year & 4 months after \\
\hline II & $0.094 / 0.058$ & NA & NA & NA & $0.053 / 0.036$ & NA \\
\hline RQ & $0.89 / 0.02$ & $0.93 / 0.02$ & $0.92 / 0.01$ & $0.93 / 0.02$ & $0.93 / 0.01$ & $0.93 / 0.01$ \\
\hline Pounds & $267.1 / 55.9$ & $259.8 / 54.3$ & $253.1 / 54.4$ & $245.8 / 54.8$ & $243.1 / 53.2$ & $240.7 / 53.1$ \\
\hline Kg & $121.3 / 25.3$ & $118.1 / 24.6$ & $115.0 / 24.8$ & $111.7 / 25.0$ & $110.6 / 24.2$ & $109.5 / 24.0$ \\
\hline BMI & $42.1 / 7.8$ & $40.9 / 7.5$ & $40.0 / 7.4$ & $38.8 / 7.7$ & $38.5 / 8.1$ & $38.0 / 8.1$ \\
\hline TC & $261.9 / 35.0$ & $247.9 / 36.1$ & $234.3 / 30.1$ & $214.4 / 30.9$ & $191.9 / 32.9$ & $182.8 / 29.6$ \\
\hline LDL & $186.2 / 37.1$ & $174.2 / 36.4$ & $163.1 / 31.2$ & $143.4 / 30.4$ & $121.8 / 33.6$ & $111.8 / 31.1$ \\
\hline HDL & $39.4 / 6.3$ & $40.8 / 5.6$ & $41.4 / 5.2$ & $44.4 / 5.8$ & $46.5 / 4.8$ & $47.8 / 3.9$ \\
\hline TC/HDL & $6.9 / 1.7$ & $6.2 / 1.4$ & $5.8 / 1.1$ & $4.9 / 1.0$ & $4.2 / 0.8$ & $3.9 / 0.8$ \\
\hline VLDL & $36.3 / 5.2$ & $32.9 / 5.4$ & $29.7 / 4.7$ & $26.6 / 4.1$ & $23.6 / 3.3$ & $23.2 / 2.9$ \\
\hline TG & $181.4 / 26.1$ & $164.3 / 26.8$ & $148.6 / 23.7$ & $133.1 / 20.3$ & $118.1 / 16.5$ & $116.0 / 14.3$ \\
\hline TG/HDL & $4.7 / 1.1$ & $4.1 / 1.0$ & $3.7 / 0.8$ & $3.1 / 0.7$ & $2.6 / 0.5$ & $2.4 / 0.4$ \\
\hline CRP & $0.54 / 0.54$ & $0.30 / 0.23$ & $0.18 / 0.09$ & $0.17 / 0.07$ & $0.13 / 0.08$ & $0.16 / 0.09$ \\
\hline IL-6 & $6.02 / 3.58$ & $5.33 / 2.85$ & $4.40 / 2.47$ & $3.78 / 2.10$ & $3.33 / 2.04$ & $3.54 / 1.98$ \\
\hline Hcy & $13.4 / 6.5$ & $12.2 / 5.4$ & $9.8 / 3.1$ & $8.5 / 2.6$ & $7.5 / 1.7$ & $7.9 / 1.9$ \\
\hline Fib & $332.6 / 63.5$ & $328.8 / 55.4$ & $322.9 / 41.7$ & $319.8 / 33.6$ & $310.4 / 26.7$ & $319.8 / 17.8$ \\
\hline Lp(a) & $24.9 / 11.8$ & $22.5 / 10.8$ & $19.8 / 9.0$ & $16.5 / 7.2$ & $14.7 / 6.3$ & $14.0 / 6.1$ \\
\hline
\end{tabular}

Table 3b: 1500-1600 kcal/day low to moderate fat diet with vitamin supplement*.

* values are presented as mean/standard deviation. 
The Weight, Inflammation, Diet and Heart (WIDtH) Study Looking at Potential Primary Prevention of Coronary Artery Disease

\begin{tabular}{|c|c|c|c|c|c|c|}
\hline & Baseline & $\mathbf{6}$ weeks & $\mathbf{3}$ months & $\mathbf{6}$ months & $\mathbf{1}$ year & 4 months after \\
\hline II & $0.081 / 0.059$ & NA & NA & NA & $0.119 / 0.079$ & NA \\
\hline RQ & $0.90 / 0.02$ & $0.88 / 0.02$ & $0.86 / 0.01$ & $0.85 / 0.02$ & $0.84 / 0.01$ & $0.93 / 0.01$ \\
\hline Pounds & $272.1 / 53.5$ & $261.4 / 53.4$ & $254.5 / 53.9$ & $250.0 / 53.5$ & $245.7 / 53.0$ & $250.0 / 53.8$ \\
\hline Kg & $123.5 / 24.2$ & $118.8 / 24.3$ & $115.9 / 24.6$ & $113.6 / 24.3$ & $111.8 / 24.0$ & $113.7 / 24.3$ \\
\hline BMI & $42.7 / 7.0$ & $41.0 / 7.0$ & $40.0 / 7.0$ & $39.3 / 7.0$ & $38.6 / 6.9$ & $39.2 / 7.1$ \\
\hline TC & $287.8 / 38.7$ & $274.7 / 36.9$ & $259.4 / 30.9$ & $269.1 / 30.6$ & $280.5 / 28.3$ & $280.5 / 26.4$ \\
\hline LDL & $206.5 / 38.7$ & $195.1 / 37.7$ & $180.9 / 33.1$ & $188.1 / 31.1$ & $198.3 / 28.0$ & $204.4 / 27.4$ \\
\hline HDL & $43.0 / 6.1$ & $43.2 / 5.3$ & $43.3 / 4.9$ & $43.7 / 5.4$ & $42.5 / 3.8$ & $42.0 / 3.0$ \\
\hline TC/HDL & $6.8 / 1.1$ & $6.4 / 1.1$ & $6.1 / 0.9$ & $6.2 / 0.9$ & $6.6 / 0.8$ & $6.7 / 0.7$ \\
\hline VLDL & $38.4 / 6.3$ & $36.4 / 5.7$ & $35.2 / 5.4$ & $37.1 / 5.3$ & $39.6 / 5.5$ & $39.1 / 9.6$ \\
\hline TG & $192.1 / 31.4$ & $181.8 / 28.6$ & $175.9 / 26.8$ & $185.7 / 26.7$ & $197.9 / 27.6$ & $195.4 / 48.0$ \\
\hline TG/HDL & $4.6 / 1.1$ & $4.3 / 0.9$ & $4.1 / 0.7$ & $4.3 / 0.7$ & $4.7 / 0.7$ & $4.7 / 1.1$ \\
\hline CRP & $1.07 / 1.79$ & $1.00 / 1.59$ & $1.34 / 1.54$ & $1.19 / 1.37$ & $1.26 / 0.59$ & $0.80 / 0.42$ \\
\hline IL-6 & $6.79 / 4.34$ & $8.14 / 3.76$ & $10.58 / 3.72$ & $10.56 / 2.50$ & $10.56 / 2.49$ & $9.70 / 3.10$ \\
\hline Hcy & $16.4 / 10.6$ & $17.3 / 9.7$ & $20.4 / 12.7$ & $21.9 / 11.5$ & $23.0 / 10.9$ & $20.4 / 10.1$ \\
\hline Fib & $311.0 / 62.7$ & $317.4 / 61.8$ & $330.1 / 56.8$ & $337.0 / 55.1$ & $340.8 / 52.2$ & $339.1 / 49.9$ \\
\hline Lp(a) & $24.3 / 10.9$ & $24.9 / 10.7$ & $26.3 / 10.8$ & $27.6 / 11.2$ & $29.9 / 11.8$ & $28.7 / 11.6$ \\
\hline
\end{tabular}

Table 4a: 1500-1600 kcal/day low carbohydrate diet without vitamin supplement*.

\begin{tabular}{|c|c|c|c|c|c|c|}
\hline & Baseline & $\mathbf{6}$ weeks & $\mathbf{3}$ months & $\mathbf{6}$ months & 1 year & 4 months after \\
\hline II & $0.066 / 0.061$ & $\mathrm{NA}$ & $\mathrm{NA}$ & $\mathrm{NA}$ & $0.116 / 0.094$ & NA \\
\hline RQ & $0.90 / 0.02$ & $0.87 / 0.02$ & $0.86 / 0.02$ & $0.85 / 0.02$ & $0.83 / 0.02$ & $0.89 / 0.02$ \\
\hline Pounds & $285.8 / 48.9$ & $269.0 / 51.7$ & $259.1 / 52.5$ & $248.3 / 47.4$ & $244.3 / 47.7$ & $249.0 / 38.9$ \\
\hline Kg & $129.9 / 22.1$ & $122.4 / 23.4$ & $117.7 / 23.9$ & $112.9 / 21.5$ & $111.1 / 21.8$ & $113.3 / 17.7$ \\
\hline BMI & $43.6 / 6.7$ & $42.3 / 8.2$ & $39.6 / 7.4$ & $38.1 / 6.8$ & $37.3 / 6.8$ & $38.1 / 5.4$ \\
\hline TC & $286.7 / 45.3$ & $277.8 / 42.1$ & $276.2 / 39.3$ & $288.7 / 43.6$ & $315.1 / 38.1$ & $315.7 / 37.5$ \\
\hline LDL & $203.8 / 45.0$ & $197.5 / 42.4$ & $195.8 / 39.7$ & $206.1 / 43.2$ & $228.3 / 38.5$ & $229.1 / 36.6$ \\
\hline HDL & $41.4 / 7.2$ & $40.6 / 5.3$ & $40.8 / 4.1$ & $40.8 / 3.4$ & $40.1 / 3.2$ & $41.6 / 2.7$ \\
\hline TC/HDL & $7.2 / 2.2$ & $7.0 / 1.9$ & $6.9 / 1.6$ & $7.2 / 1.5$ & $7.9 / 1.4$ & $7.7 / 1.3$ \\
\hline VLDL & $41.7 / 9.0$ & $39.6 / 8.2$ & $39.5 / 7.3$ & $41.8 / 6.7$ & $46.6 / 8.3$ & $44.8 / 7.0$ \\
\hline TG & $208.4 / 45.1$ & $198.0 / 40.8$ & $197.6 / 36.5$ & $209.2 / 33.6$ & $232.9 / 41.7$ & $224.2 / 35.1$ \\
\hline TG/HDL & $5.2 / 1.3$ & $5.0 / 1.3$ & $4.9 / 1.1$ & $5.2 / 1.1$ & $5.9 / 1.2$ & $5.4 / 1.1$ \\
\hline CRP & $0.96 / 0.97$ & $0.79 / 0.48$ & $0.68 / 0.23$ & $0.74 / 0.15$ & $0.62 / 0.21$ & $0.42 / 0.21$ \\
\hline IL-6 & $5.28 / 2.86$ & $5.67 / 2.67$ & $6.67 / 3.40$ & $7.54 / 3.20$ & $7.23 / 2.62$ & $6.42 / 2.45$ \\
\hline Hcy & $15.1 / 8.0$ & $15.4 / 6.8$ & $14.8 / 6.5$ & $14.4 / 5.6$ & $15.1 / 4.6$ & $14.5 / 4.5$ \\
\hline Fib & $332.6 / 67.3$ & $340.8 / 65.0$ & $351.9 / 61.5$ & $360.6 / 50.2$ & $372.4 / 47.5$ & $373.5 / 48.1$ \\
\hline Lp(a) & $20.2 / 12.0$ & $21.9 / 11.0$ & $22.9 / 10.7$ & $24.0 / 11.0$ & $25.5 / 11.1$ & $25.8 / 10.5$ \\
\hline
\end{tabular}

Table 4b: 1500-1600 kcal/day low carbohydrate diet with vitamin supplement*.

* values are presented as mean/standard deviation. 
pounds/121.3 kilograms. After one year their weight dropped by $9 \%$ losing 24.0 pounds/10.7 kilograms. Their average BMI dropped from 42.1 to 38.5. After an additional four months, continued weight loss was noticed which was significant $(\mathrm{p}<0.05)$ for the group without supplement use and approached significance for those taking the supplement.

Finally, those on the low carbohydrate diet without supplementation lost an average of 26.4 pounds/11.7 kilograms. This represented a $9.7 \%$ weight loss with an average decrease in BMI from 42.7 to 38.6. This was not ( $\mathrm{p}=\mathrm{NS}$ ) significant. Four months later, there was a 4.3 pound/1.9 kilogram weight gain. The group following the low carbohydrate diet with supplement showed an average weight loss of 41.5 pound/18.8 kilogram, which was statistically $(p<0.01)$ significant. During that time the average BMI dropped from 43.6 to 37.3. Four months later there was a weight increase of 4.7 pounds/2.2 kilograms. BMI increased during this time from 37.3 to 38.1

\section{Depository (lipid) variables}

Tables' $2 \mathrm{a}$ to $4 \mathrm{~b}$ show the results of changes in lipids for each of the groups throughout the study. Subjects on the vegetarian diet without supplement showed an average decrease in total cholesterol of $48.6 \%$, and a $39.8 \%$ decrease in LDL during the first 12 months. These changes were statistically $(\mathrm{p}<0.001 / \mathrm{p}<0.001)$ significant and continued to improve during the four month follow up. Similarly, the VLDL and TG levels decreased by an average of $14.0 \%$ and $70.1 \%$ respectively following 12 months of dietary change. These results were significant $(\mathrm{p}<0.001 / \mathrm{p}<0.001)$ respectively. The HDL levels increased by an average of $5.3 \%$ during the first 12 months while the TC/HDL levels dropped from 6.0 to 4.3 . This increase in HDL $(p<0.01)$ and decrease in TC/HDL $(p<0.001)$ is significant. Further improvements in lipids continued to be present 4 months later.

Individuals on the vegetarian diet who were taking the supplement decreased their TC levels by an average of $71.6 \%$ during the first 12 months. Similarly, their LDL levels fell an average of $62.7 \%$. Both changes were significant at the $\mathrm{p}<0.001$ level. During this same time the VLDL and TG levels decreased by $13.6 \%$ and $68.1 \%$ respectively. These changes were significant at $\mathrm{p}<0.001$ for each. These decreases continued through the four month follow up period. HDL levels increased by an average of $10.8 \%$ (4.7) during the 12 months of dietary change. This was significant $(p<0.005)$ and remained significant 4 months later. The TC/HDL levels decreased from 6.2 to 4.0 during this 12 month period, which was a statistical $(\mathrm{p}<0.001)$ improvement and remained this way 4 months later.
The TC and LDL levels dropped by an average of $84.7 \mathrm{mg} / \mathrm{dl}$ and $75.2 \mathrm{mg} / \mathrm{dl}$ respectively on the low to moderate fat diet without supplement. These changes are each statistically significant at the $p<0.001$ level. Further improvement was seen four months later as shown in table 3a. This same group showed a $37 \%$ average reduction in VLDL at 1 year. This change was significant at $\mathrm{p}<0.001$. This decrease continued and was $41.8 \%$ below baseline values 4 months after the study. The TG level began at an average of 197.4 $\mathrm{mg} / \mathrm{dl}$ and had decreased by $36.9 \%$ by the end of one year. This was significant at the $\mathrm{p}<0.001$ level and continued to show improvement 4 months later with a decrease of $41.7 \%$. Participants on this diet showed an average increase in HDL levels of $4.9(\mathrm{p}<0.005)$ and reduction in average TC/HDL from 6.2 to $3.8(\mathrm{p}<0.001)$ during the first 12 months. These changes continued to show improvement four months later as shown in table $3 a$.

On the low to moderate fat diet with supplement, TC and LDL levels decreased from an average of 261.9 to $191.9(\mathrm{p}<0.001)$ and 186.2 to 121.8 ( $\mathrm{p}<0.001) \mathrm{mg} / \mathrm{dl}$ respectively. Continued improvement was seen 4 months later when the average TC and LDL were 182.8 and $111.8 \mathrm{mg} / \mathrm{dl}$. The VLDL and TG levels decreased by $35 \%$ ( $p<0.001)$ and $34.9 \%(p<0.001)$ during the first 12 months of the study and $36.1 \%$ and $36.1 \%$ respectively four months later when compared with baseline values. The HDL increased by $18 \%$ ( $\mathrm{p}<$ 0.001 ) during the first 12 months and by $21.3 \%$ by the 4 month assessment after the diet. The TC/HDL ratio decreased from an average of 6.9 at baseline to $4.2(p<0.001)$ at 12 months and 3.9 four months later.

During the twelve month low carbohydrate diet without supplement individuals TC and LDL levels decreased during the first three months. The average TC decreased from 287.8 to $259.4 \mathrm{mg} / \mathrm{dl}$ during this time, which was significant $(\mathrm{p}<0.025)$; however, during the next nine months the average TC actually increased to an average of $280.5 \mathrm{mg} / \mathrm{dl}$. It remained at this level during the four month reassessment period following the diet. The LDL had an average value of 206.5 at baseline with a nadir of $180.9(p<0.05)$ at 3 months The LDL value then increased during the remaining 9 months to an average value of $198.3 \mathrm{mg} / \mathrm{dl}$. Four months later the average LDL was $204.4 \mathrm{mg} / \mathrm{dl}$. VLDL and TG levels initially decreased for the first three months; however, this was not significant and by the end of the study the VLDL and TG levels were $3.1 \%$ and $3.0 \%$ above baseline respectively. HDL levels showed a slight decrease from an average of $43.0 \mathrm{mg} / \mathrm{dl}$ to $42.5 \mathrm{mg}$ after one year on the diet. During this same time the TC/HDL ratio showed a mild drop from 6.8 to 6.6. This represented a $2.9 \%$ decrease. These changes in HDL and TC/HDL were not ( $p=N S$ ) significant. 
Those on the low carbohydrate diet with supplement showed an initial non-significant decrease in TC during the first three months. By the end of the twelfth month the TC increased from an average of 286.7 to $315.1 \mathrm{mg} / \mathrm{dl}$, which represented a statistical $(\mathrm{p}<0.05)$ increase. The LDL levels began at an average of $203.8 \mathrm{mg} / \mathrm{dl}$. This decreased non-statistically to 195.8 after three months of dieting and nine months later had increased to an average of $228.3 \mathrm{mg} /$ $\mathrm{dl}$, which approached but did not reach significance. The VLDL and TG levels initially decreased during the first three months. By the end of the twelfth month the VLDL level was $11.8 \%$ ( $p=N S$ ) above baseline while TG was $11.8 \%$ ( $p=N S$ ) above baseline.

\section{Insulin resistance $(\mathrm{tg} / \mathrm{hdl})$ changes [9]}

Changes in TG/HDL values are shown in tables' 2a to $4 \mathrm{~b}$. Levels of TG/HDL decreased by an average of $43.5 \%$ (p < 0.001) when people consumed a vegetarian diet without supplement. When supplement was added there was a $40.0 \%$ ( $p<0.001$ ) average reduction in TG/HDL. On the low to moderate fat diet without supplement, the average TG/HDL ratio decreased by $43.5 \%(\mathrm{p}<0.001)$. Individuals receiving supplement while following the low to moderate fat diet showed an average reduction of $44.7 \%(\mathrm{p}<0.001)$ in TG/HDL ratio. Those following a low carbohydrate diet had an average increase of $2.2 \%$ ( $\mathrm{p}=\mathrm{NS}$ ) without supplement and a $13.5 \%$ increase $(p=N S)$ with the supplement.

\section{Inflammothrombotic variables}

Results for these variables are shown in tables $2 \mathrm{a}$ to $4 \mathrm{~b}$. C-reactive protein decreased by $65.4 \%$, which approached $(p=N S)$ but did not reach significance when individuals followed a vegetarian diet without supplementation. When supplementation was added, the CRP level decreased by $88.0 \%(p<0.025)$ after 12 months. The CRP value was statistically higher $(p<0.05)$ at the beginning of the study in this group of individuals than in the group following the low to moderate fat diet taking supplementation. In this later group, the CRP values fell by an average of $75.9 \%(p<0.005)$ during the twelve months on their diet, while the group on the low to moderate fat diet without supplementation showed an average 87.3\% $(\mathrm{p}<0.025)$ reduction in CRP values. Those on the low carbohydrate diet without supplementation had an average increase of $17.8 \%$ ( $\mathrm{p}=\mathrm{NS}$ ) in CRP while those on the low carbohydrate diet with supplementation had a $35.4 \%$ reduction $(p=N S)$ in CRP.

When people followed a vegetarian diet without supplement the IL-6 levels fell by an average of $50.2 \%(\mathrm{p}<0.01)$ during the first twelve months with a further decrease to a $60.0 \%$ drop from baseline values noted during the 4 month follow up assessment. When the vegetarian diet was combined with the supplement, the IL-6 levels decreased by $39.1 \%$ ( $p<0.01$ ) after 12 months with continued reduction ( $42.7 \%$ ) by the four month follow up after the 1 year dietary regimen. The low to moderate fat diet without supplement showed a non-statistical reduction (35.0\%) in IL-6 while those following the same diet with supplement showed an average reduction of $44.7 \%(p<0.01)$ following 12 months of dietary change. After 12 months on a low carbohydrate diet without supplement, the average IL-6 levels increased by $55.5 \%$ ( $p<0.005)$. Those on the low carbohydrate diet with supplement showed a less pronounced increase in IL- 6 of $36.9 \%$ ( $p<0.05$ ) following 12 months on the diet. Both groups (with and without supplement) showed reduction in IL-6 levels following cessation of the low carbohydrate diet.

Homocysteine levels decreased by an average of $27.1 \%(p=N S)$ on the vegetarian diet without supplement and returned to baseline values when subjects were reassessed four months later. Those following the vegetarian diet with supplement showed a $34.5 \%$ (p $<0.01$ ) average reduction in homocysteine after one year, which persisted with further improvement four months later. The low to moderate fat diet without supplement resulted in a $42.5 \%$ average reduction in homocysteine levels $(\mathrm{p}<0.005)$. When the supplement was added there was a $44.0 \%$ reduction $(\mathrm{p}<0.001)$ in homocysteine at one year. The low carbohydrate diet without supplement showed an average increase in homocysteine of $40.2 \%$, which approached but did not reach statistical significance. This increase in homocysteine was attenuated with the addition of the supplement, which was associated with no change in homocysteine over the 12 month diet.

Fibrinogen levels showed a mild non-statistical increase for people following vegetarian diets with supplement, along with those who followed a low to moderate fat diet without supplement and the low carbohydrate diet without supplement. Those on the vegetarian diet without supplement showed a non-statistical decrease in fibrinogen. Those on the low to moderate fat diet taking the supplement showed an average $6.7 \%(\mathrm{p}=\mathrm{NS})$ reduction in fibrinogen. People following the low carbohydrate diet taking the supplement showed an average increase of $12.0 \%(p<0.05)$ in fibrinogen.

Finally, Lp(a) values decreased by an average of $23.3 \%(\mathrm{p}=\mathrm{NS})$ on the vegetarian diet without supplement and by $18.8 \%$ ( $\mathrm{p}=\mathrm{NS}$ ) with supplement. Those following the low to moderate fat diet showed an average reduction of $34.2 \%(p<0.01)$ without supplement and $41.0 \%$ ( $p<0.005)$ with the supplement. The low carbohydrate diet was associated with a $23.0 \%(\mathrm{p}=\mathrm{NS})$ average increase without supplement and a $26.2 \%$ ( $p=N S$ ) increase with the supplement. 


\section{Discussion}

There were no statistical differences between groups at the beginning of the study with the exception of CRP, which was higher for those on the vegetarian diet with supplement than for those on the low to moderate fat diet who received the supplement. The reason for this difference is unclear but did not appear to affect outcomes.

Participants in the study reported no difficulty followed their recommended dietary programs for one year. Dietary change was noted during the initial 6 week assessment with the detection of urinary ketones among those on the low carbohydrate diets only. Similarly changes in $\mathrm{RQ}$ values for each group were consistent with their respective dietary regimen. As with most dietary regimens, the initial weight loss appears the most impressive with slower weight loss occurring later in the year. This is to be expected since caloric intake was not reduced throughout the study resulting in less disparity between actual caloric requirements and that consumed as weight loss occurred. Following 12 months of dietary change, the vegetarian group appeared to modify their diets only slightly during the 4 month follow up with either no increase in $\mathrm{RQ}$ or a reduction. The change in RQ among those on the vegetarian diet with supplement may reflect a change in behavior present with discontinuation of the supplement, while those who did not receive the supplement showed no change in RQ. No change in eating behavior was seen for those consuming the low to moderate fat diet regardless of whether they had been receiving the supplement. Those on the low carbohydrate diet changed their eating patterns considerably by the 4 month re-evaluation session, with increases in RQ approximating that of the other groups. This increase in RQ was associated with an increase in weight for those on the low carbohydrate group. The individuals following the vegetarian diet showed stabilization of weight while those on the low to moderate fat regimen showed persistent weight loss even after the first 12 months of the study was completed. These findings indicate that the less extreme the diet, the greater the likelihood of continued weight loss success.

Lipids initially decreased in all groups consistent with the reduction in caloric intake of those in the study. Those following both the vegetarian and low to moderate fat diets showed continued reduction in TC, LDC, VLDL and TG levels that remained even after the study was completed. Those following the low carbohydrate diet showed reductions in these same lipids during the first three months of the study when the decrease in calorie intake could have resulted in the utilization of lipid and fatty acid substrates as a source of energy. After this brief period of time, the primary source of caloric intake, confirmed by RQ and urine ketones, was fat. Subsequently, this source of fatty acids appears to have been more than adequate to increase lipid levels as shown in tables $4 \mathrm{a}$ and $4 \mathrm{~b}$.

Each group participated in the same exercise regimen and there were no confounders (e.g. Estrogen use) with roughly equal numbers of women in each group and roughly equal numbers of men and women in the study. Those on the vegetarian and low to moderate fat diets showed similar increases in HDL, which was not influenced by the supplement. There was essentially no change in HDL among those individuals following the low carbohydrate diet. The resultant changes which one would expect to see for TC/HDL ratios, given the above findings, was noted with those on both the vegetarian and low to moderate fat diets showing reductions which were statistically $(\mathrm{p}<0.001)$ significant. There was a mild insignificant decrease in TC/HDL for those on the low carbohydrate diet without supplement and a mild insignificant increase in TC/HDL for those taking the supplement. The reason for this increase is unclear but remained insignificant.

Insulin resistance as determined using TG/HDL ratio improved significantly $(\mathrm{p}<0.001)$ for those on the vegetarian and low to moderate fat diets. This was expected since the caloric intake (a major component of insulin resistance with adult onset diabetes mellitus) was taken into account through caloric restriction. Using TG/HDL, insulin resistance increased on the low carbohydrate diet, with improvement noted during the 4 month follow up period among those who had been receiving supplement. While it is not certain why this occurs, it has been reported previously [22-25].

Evidence of inflammatory changes was seen most directly through the measurement of CRP and IL-6. The CRP level decreased significantly among those on the low to moderate fat diet and for those on the vegetarian diet receiving supplement. The decrease in CRP was present but less significant for vegetarians not receiving the supplement. Changes in CRP were mixed on the low carbohydrate diet with improvement in this group also associated with the use of the supplement suggesting a potential anti-inflammatory effect from the supplement. A slightly different response was seen when IL- 6 was measured. Those on the supplement who were following the vegetarian or low to moderate fat diet showed significant $(\mathrm{p}<0.01)$ reductions in IL- 6 as did the vegetarian group who did not take the supplement. Those following the low to moderate fat diet and did not take the supplement showed a reduction in IL6, which did not reach statistical significance. An increase in IL-6 was seen for those on the low carbohydrate diet, which was less pronounced for the group taking the supplement. 
Homocysteine levels changed only slightly for those on the vegetarian diet without supplement. Those on the low carbohydrate diet not taking the supplement showed an increase in Hcy, which did not reach statistical significance. Both the vegetarian group and the low carbohydrate group showed better results when the supplement was added. For those on the low carbohydrate group taking the supplement there was essentially no change until they stopped following the diet. Individuals on the vegetarian diet showed significant reductions $(\mathrm{p}<0.01)$ in Hcy. Those on the low to moderate fat diet showed reductions in Hcy without the supplement ( $p<0.005)$, which was more pronounced $(p<0.001)$ when the supplement was taken. These findings demonstrate a significant role for the supplement among people on the vegetarian and low carbohydrate diets. Presumably due to vitamin B12 deficiency in the vegetarian group and the increase in methionine and decrease in folate and possibly B6 for those eating a low carbohydrate diet.

Fibrinogen levels were essentially unchanged in this study with mild decreases noted only for those on either a vegetarian of low to moderate fat diet. These changes were not significant and remained essentially unchanged during the four month follow up. Differences in exercise and hormone therapy were eliminated in this study.

Lipoprotein (a) showed a slight improvement on the vegetarian diet and a slight increase on the low carbohydrate diet. Only those on the low to moderate fat diet showed significant improvements in the Lp(a) levels which was most pronounced with the supplement.

Basal metabolic index followed the pattern discussed for weight loss above with an average reduction in BMI of 9.6 to $14.4 \%$. This success was maintained for those on the vegetarian diet and continued to show improvement on the low to moderate fat diet. This continued improvement on the low to moderate fat diet and the persistence of RQ on this diet demonstrated not only an ability to lose weight on the low to moderate fat diet but also the ability to continue to lose weight as needed. Those on the vegetarian diet were able to maintain their weight following cessation of the study. Further weight loss might be achieved on a longer term low to moderate fat diet following an initial vegetarian diet. Initially, the low carbohydrate diet showed more weight loss, although the difference ( $p=N S$ ) between the low carbohydrate diet and the low to moderate fat or the vegetarian diet was minimal. After the 12 months on the low carbohydrate diet, individuals showed a change in dietary pattern reflected by increased RQ and absence of ketones in the urine. There was an average weight gain of 4-5 pounds/ 2 kilograms during the next 4 months after people discontinued the low carbohydrate diet.
Despite these differences in lipids, inflammothrombotic factors and weight changes, coronary blood flow did not improve in all groups supporting the idea that weight alone is not the only determinant of heart disease. Coronary blood flow decreased among individuals following the low carbohydrate diet. This worsening of blood flow was less pronounced when the supplement was taken. Those on the vegetarian diet showed improvement in coronary blood flow. This change was not significant. Improvement in coronary blood flow was also seen with the low to moderate fat diet which was significant $(\mathrm{p}<0.025)$ when the supplement was also taken.

The results of this study in individuals who are obese (BMI>30) demonstrate that weight loss can be achieved through a variety of diets. However weight loss does not persist with all diets and the use of the supplement had several potential benefits depending upon the diet being followed. Numerous CVDRFs are influenced as a result of these dietary changes, which may adversely affect coronary blood flow.

\section{Conclusion}

One size does not fit all! The type of diet one follows should reflect the results one is trying to obtain. In the absence of demonstrable heart disease (angina or angina equivalent symptoms) weight loss can be achieved as long as caloric restriction is implemented. In this study, exercise was a mandatory component, which was easily achieved by those involved. Exercise does not need to be equivalent to training for the Olympics, rather, 30 minutes of walking, bicycling or swimming three times a week was adequate to achieve the desired results including weight loss and reduction of CVDRFs as long as a vegetarian or low to moderate fat diet was followed. Initial improvement in lipids on the low carbohydrate diet was followed by worsening of these during the months that followed. Individuals on both a vegetarian and low carbohydrate diet benefit from the addition of the folate, B6, B12 supplement. A reduction in insulin resistance was seen in addition to improvement in coronary blood flow on both the vegetarian and low to moderate fat diet. This was not seen on the low carbohydrate diet. Finally, while obesity is presenting as an ever increasing risk factor for heart disease, diabetes, hypertension and certain forms of cancer, the diet one uses to lose weight may have adverse effects regardless of the effect on weight itself.

Potential COI: Ischemic Index is the precursor to FMTVDM issued to primary author with

All figures and data are reproduced with permission of the primary author. 


\section{Bibliography}

1. Ornish D., et al. "Can lifestyle changes reverse coronary artery heart disease?" Lancet 336.8708 (1990): 129-133.

2. Fleming RM., et al. "Treating hyperlipidemia in the elderly". Angiology 46.12 (1995): 1075-1083.

3. Fleming RM., et al. "Assessing the independent effects of dietary counseling and hypolipidemic medications on serum lipids". Angiology 47.9 (1996): 831-840.

4. Barnard RJ. "Effects of life-style modification on serum lipids". Archives of internal medicine 151.7 (1991): 1389-1394.

5. Fleming RM. “The Pathogenesis of Vascular Disease”. In: Chang JC, ed. The Textbook of Angiology, New York, NY: Springer-Verlag (1999): 787-798.

6. Fleming RM and Boyd LB. "Reversing heart disease in the new millennium. The Fleming Unified Theory". Angiology 51.8 (2000): 617-629.

7. Fleming RM. "The effect of high-, moderate-. And low-fat diets on weight loss and cardiovascular risk factors". Preventive Cardiology 5.3 (2002):110-118.

8. Fleming RM. "The effect of high protein diets on coronary blood flow". Angiology 51.10 (2000): 817-826.

9. Westman EC., et al. "Effect of 6-month adherence to a very low carbohydrate diet program". American Journal of Medicine 113.1 (2002): 30-36.

10. Fleming RM. "Caloric intake, not carbohydrate or fat consumption, determines weight loss". American Journal of Medicine 114.1 (2003):78.

11. Foster GD., et al. "A randomized trial of a low-carbohydrate diet for obesity". New England Journal of Medicine 348.2 (2003): 2082-2090.

12. Samaha FF., et al. "A low-carbohydrate as compared with a low-fat diet in severe obesity". New England Journal of Medicine 348 (2003): 2074-2081.

13. LaRosa JC., et al. "Effects of high-protein, low-carbohydrate dieting of plasma lipoproteins and body weight". Journal of the American Dietetic Association 77.3 (1980): 264-270.

14. Vogel RA., et al. "Effect of a single high fat meal on endothelial function in healthy subjects". American Journal of Cardiology 79.3 (1997): 350-354.
15. Esposito K., et al. "Effect of weight loss and lifestyle changes on vascular inflammatory markers in obese women". Journal of the American Medical Association 289.14 (2003): 1799-1804.

16. Luscombe ND., et al. "Effect of a high-protein, energy-restricted diet on weight loss and energy expenditure after weight stabilization in hyperinsulinemic subjects". International journal of obesity and related metabolic disorders 27.5 (2003): 582-590.

17. Fernandez-Real JM and Ricart W. "Insulin resistance and chronic cardiovascular inflammatory syndrome". Endocrine Reviews 24.3 (2003): 278-301.

18. De Pergola G and Pannacciulli N. "Coagulation and fibrinolysis abnormalities in obesity". Journal of Endocrinological Investigation 25.10 (2002): 899-904.

19. Rallidis LS., et al. "Dietary alpha-linolenic acid decreases Creactive protein, serum amyloid A and Interleukin- 6 in dyslipidaemic patients". Atherosclerosis 167.2 (2003): 237-242.

20. Fleming RM and Boyd LB. "High-dose dipyridamole and gated sestamibi SPECT imaging provide diagnostic resting and stress ejection fractions useful for predicting extent of coronary artery disease". Angiology 53.4 (2002): 415-421.

21. Snedecor and Cochran Statistical Methods Sixth edition, The Iowa State University Press, Ames, IA, USA (1979): 549.

22. Anderson JW and Herman RH. "Effects of carbohydrate restriction on glucose tolerance of normal men and reactive hypoglycemic patients". American Journal of Clinical Nutrition 28.7 (1975): 748-755.

23. Simpson RW., et al. "Improved glucose control in maturity-onset diabetes treated with high-carbohydrate-modified fat diet". British medical journal 1.6180 (1979): 1753-1756.

24. Simpson HC., et al. "Digestible carbohydrate-an independent effect on diabetic control in type 2 (non-insulin-dependent) diabetic patients?". Diabetologia 23.3 (1982): 235-239.

25. Ward GM., et al. "Insulin receptor binding increased by high carbohydrate low fat diet in non-insulin-dependent diabetes". European Journal of Clinical Investigation 12.2 (1982): 93-96.

\section{Volume 3 Issue 7 July 2019}

(C) All rights are reserved by Richard M Fleming., et al. 